\title{
40. THE STUDY OF THE MILKY WAY INTEGRATED SPECTRUM AND THE SPIRAL STRUCTURE OF THE GALAXY
}

\author{
E. B. KOSTJA KOVA
}

Moscow University, Moscow, U.S.S.R.

The integrated spectrum of the Milky Way can give some information on the composition and large-scale structure of the Galaxy.

A study of the integrated spectrum of the Milky Way was carried out by the author in both hemispheres during several years (Kostjakova, 1952, 1954, 1958, 1963, 1964, 1965; Sharov and Kostjakova, 1967).

The absolute spectrophotometric gradients $(\Phi)$ and temperatures $\left(T_{\mathrm{c}}\right)$ of 20 bright Milky Way fields studied are shown in Figure 1 (filled circles). The solid curve indicates the colour distribution of the Milky Way with the galactic longitude. We can see that over a large part of the Milky Way strip $\left(120^{\circ}<l^{\mathrm{II}}<320^{\circ}\right)$ the spectrophotometric temperature is fairly high, of about $7000-8000 \mathrm{~K}$. Beginning at $l^{\mathrm{II}}=100^{\circ}-110^{\circ}$, it declines gradually toward the direction of the galactic centre, reaching there its minimum value of about $4000 \mathrm{~K}$. The pattern appears to be an asymmetric one: in the northern Milky Way the decline in temperature sets in at a greater angular distance than in the south.

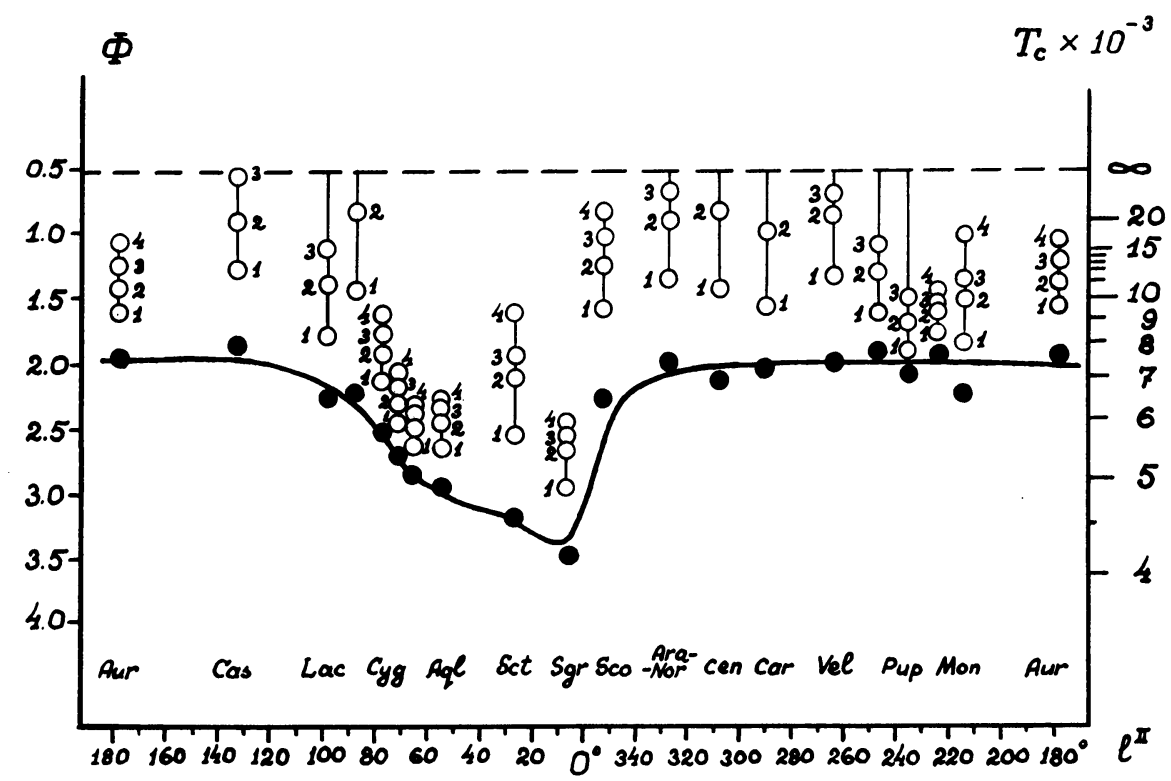

Fig. 1. The spectrophotometric temperature, $T_{\mathrm{c}}$, of the Milky Way, plotted against galactic longitude, $l^{\mathrm{II}}$. 
The results obtained were corrected for the interstellar absorption by the method of Parenago (1945), with the data of $a_{0}$ (absorption per $\mathrm{kpc}$ ), according to Sharov (1963). The correction procedure is illustrated also in Figure 1, where the open circles with numbers show the values of $T_{\mathrm{c}}$, obtained for four assigned distances: $1,2,3 \mathrm{kpc}$, and $r=\infty$. The results show that the observed reddening of the Milky Way toward the galactic centre is a real effect, not caused by the interstellar absorption. In the 'cool' Milky Way clouds (Sagittarius, Scutum, Aquila), with a plausible $a_{0}$ adopted, $T_{\mathrm{c}}$ varies over a comparatively small range, while the distance changes greatly. The 'hot' clouds, on the contrary, are very sensitive to the choice of distance. That enables to estimate an upper limit of the distance to individual 'hot' Milky Way clouds. Such estimates were made for several clouds.

The revealed colour distribution of the Milky Way was explained by the difference in stellar composition of the Galaxy in various directions. In regions of high temperature $\left(T_{\mathrm{c}}\right)$ the main contribution to the integrated radiation evidently comes from hot (blue) giants and supergiants, typical for spiral arm population. By decreasing the angular distance from the galactic centre, the contribution of the 'red populations' - spherical and intermediate - begins to predominate in the total radiation. Such an explanation agrees well with current ideas on the structure and stellar composition of galaxies.

The study of the integrated Milky Way spectrum, together with the upper bound estimates on distances to several clouds, led to certain conclusions as to the galactic structure in the solar neighbourhood.

(1) The colour variations in the northern Milky Way indicate that the region where 'red objects' predominate, has a large angular size. This agrees with the conclusion of Bok $(1956,1967)$ that the nuclear region of our Galaxy extends to about 5-6 kpc in radius.

(2) The progressive rise of $T_{\mathrm{c}}$ in the direction of Cygnus-Aquila may indicate an actual increase in the number of the flat subsystem objects toward the anticentre; it appears that in this direction we observe the beginning of a spiral arm.

(3) The higher (real) temperature in Scutum as compared with adjacent regions can also be explained by the assumption that in this direction we observe the beginning of an inner spiral arm. In the direction of Sagittarius the line of sight intersects this arm; the remaining radiation would be produced by the coolest stars of the spherical component. In other directions $\left(120^{\circ}-320^{\circ}\right)$ the main contribution to the integrated radiation evidently comes from flat component objects, causing a high $T_{\mathrm{c}}$ there.

(4) The asymmetry in the galactic longitude dependence of the Milky Way colour can easily be explained by an asymmetric placement of the spiral arms relative to the direction from the sun to the galactic centre.

The spiral arms outlined, agree well with the location of the Population I objects, according to the recent studies of the Galaxy by optical methods (Becker 1964; Becker and Fenkart 1963; Beer, 1961; Schmidt-Kaler 1964; Whiteoak, 1963) (see Figure 2 in Kostjakova, 1965).

A similar analysis, carried out in reference to the results of radio astronomical research, led to the conclusion that in the solar neighbourhood the spiral arms deduced 
by optical methods and those derived from radio observations, agree neither in position nor in direction. This fact still awaits its explanation.

\section{References}

Becker, W.: 1964, Z. Astrophys. 58, 202.

Becker, W. and Fenkart, R.: 1963, Z. Astrophys. 56, 257.

Beer, A.: 1961, Monthly Notices Roy. Astron. Soc. 123, 191.

Bok, B. J.: 1956, Vistas in Astronomy 2, 1522.

Bok, B. J.: 1967, Am. Scient. 55, No. 4.

Kostjakova, E. B.: 1952, Soviet Astron. Circ. No. 129.

Kostjakova, E. B.: 1954, Izv. Krym. Astrofiz. Obs. 12, 118.

Kostjakova, E. B.: 1958, Soviet Astron. Circ. No. 192, 13.

Kostjakova, E. B.: 1963, Astron. Zh. 40, 771.

Kostjakova, E. B.: 1964, Astron. Zh. 41, 505.

Kostjakova, E. B.: 1965, Astron. Zh. 42, 537.

Parenago, P. P.: 1945, Astron. Zh. 22, 129.

Schmidt-Kaler, T.: 1964, Z. Astrophys. 58, 217.

Sharov, A. S.: 1963, Astron. Zh. 40, 900.

Sharov, A. S. and Kostjakova, E. B.: 1967, Astron. Zh. 44, 98.

Whiteoak, J. B.: 1963, Publ. Astron. Soc. Pacific 75, 103. 